\title{
Surgery for Varicose Veins Caused by Atypical Incompetent Perforating Veins
}

\author{
Hitoshi Kusagawa, MD, PhD
}

Objective: To clarify the localization of and surgery for atypical incompetent perforating veins (IPVs) other than Dodd, Boyd, Cockett perforators, which have not been previously discussed.

Methods: Forty-three atypical IPVs, diagnosed by venous ultrasonography and treated surgically from January 2014 to June 2018, were analyzed from the viewpoint of localization and surgical treatment.

Results: All atypical IPVs passed through the fascia in the area between the muscle compartments in the same way as the typical IPV. The IPVs were most commonly located in the posterolateral part of the lower thigh (16), around the popliteal fossa (9), and in the posterolateral part in the lower calf (7). For 42 IPVs, surgery consisted of direct ligation and resection at the fascia level, and foam sclerotherapy was performed for 1 IPV. The blood flow of the perforating vein was blocked under the fascia in 40 IPVs, but to and fro flow at the fascia level remained in 3 IPVs.

Conclusion: Atypical IPVs causing varicose veins were most common in the posterolateral part of the lower thigh. For treatment, it is important to ligate and cut them without leaving stumps with related branches outside the fascia under precise ultrasonic observation. (This is a translation of Jpn J Vasc Surg 2018; 27: 461-466.)

Keywords: varicose vein, lower extremity, ultrasonography, surgical procedures

\section{Introduction}

Although perforator veins of the lower extremities were

Matsusaka Obta Clinic, Matsusaka, Mie, Japan

Received: July 1, 2019; Accepted: July 1, 2019

Corresponding author: Hitoshi Kusagawa, MD, PhD. Matsusaka Ohta Clinic, 993 Shimomura-cho, Matsusaka, Mie 515-0043, Japan

Tel: +81-598-29-1213, Fax: +81-598-29-1262

E-mail: kusagawa@oota-cli.jp

This is a translation of Jpn J Vasc Surg 2018; 27: 461-466.

(cc) BY-NC-SA (C2019 The Editorial Committee of Annals of Vascular Diseases. This article is distributed under the terms of the Creative Commons Attribution License, which permits use, distribution, and reproduction in any medium, provided the credit of the original work, a link to the license, and indication of any change are properly given, and the original work is not used for commercial purposes. Remixed or transformed contributions must be distributed under the same license as the original. named in 2002 by the International Union of Phlebology (UIP), ${ }^{1)}$ the names are not commonly used, and among physicians who specialize in the treatment of incompetent perforating veins (IPVs), the perforators that are considered clinically important are still referred to as Dodd, Boyd, and Cockett, which are the names of former venous surgeons. ${ }^{2)}$ In contrast, lower extremity varicose veins in other atypical positions caused by IPVs may be encountered and also be treated. In this study, their localization, presentation, symptoms, treatment, and associated results are summarized, with the importance of treatment based on accurate diagnosis along with considerations based on the literature.

\section{Patient Population and Methods}

Lower extremity varicose veins in atypical locations due to IPVs in 43 limbs diagnosed using lower extremity vein echocardiography between January 2014 and June 2018 and treated as per patients' will were analyzed. These cases accounted for approximately $2 \%$ of all patients who underwent lower extremity varicose vein surgery during the same period. The mean age of patients was $67.1 \pm 12.8$ years, 11 limbs were from men and 32 limbs were from women, and the clinical stage based on ClinicalEtiology-Anatomy-Pathophysiology classifications was C2/3/4a/4b/5/6 =26/5/8/1/1/2. Complications of deep vein lesions were found in 4 limbs $(9.3 \%)$, with C6 post thrombotic syndrome in $1 \mathrm{limb}$, deep vein reflux in $1 \mathrm{limb}$, C5 deep vein reflux in 1 limb, and C3 iatrogenic femoral vein occlusion in 1 limb. Previous surgery for saphenous vein lesions was reported in 9 limbs $(21 \%)$, including 6 vein strippings and 3 radiofrequency ablations. Simultaneous surgery of the saphenous vein was performed in 7 limbs $(17 \%)$, including 6 radiofrequency ablations and 1 vein stripping. In these patients, the skin just above the IPVs was marked, and a $<1-\mathrm{cm}$ skin incision was made to separate the IPV. On the central side, the side branches were treated to advance the separation to the fascial level, and the ligature was cut off. On the peripheral side, the side-branch varicose veins were resected. In all patients, the treatment result was postoperatively evaluated using lower extremity vein echocardiography in the first month, 
and improvement in symptoms was assessed.

\section{Results}

The localization of atypical IPVs is shown in Fig. 1. All IPVs were found between muscle compartments (Fig. 2). The most common type was posterolateral thigh perforating veins (UIP name: $5.4 .3^{1)}$ ) present on the lateral side of the biceps femoris lateral head on the posterolateral side of the lower thigh, which was observed in 16 limbs (37\%). Subsequently, there were 9 limbs with veins near the popliteal fossa (UIP name: popliteal fossa perforating veins, $4.5,21 \%$ ) and 7 limbs with posterolateral veins in the lower thigh (UIP name: lateral gastrocnemius perforating veins, part of $3.4 .2,16 \%$ ). Other findings included veins in the posterior aspect of the middle lower thigh (UIP name: intergemellar perforating veins, 3.4.3), those in the posterolateral side of the middle lower thigh (UIP name: lateral gastrocnemius perforating veins, 3.4.2), and those in the ankle flexion side (UIP name: anterior ankle perforating veins, 2.2).

Symptoms in 36 limbs without saphenous venous lesions and the total numbers of them are shown in the Table 1 . Although the primarily reported symptoms were discomfort in 25 limbs and sluggishness in 14 limbs, these resolved or improved postoperatively in all patients.

In lower extremity vein echocardiography performed

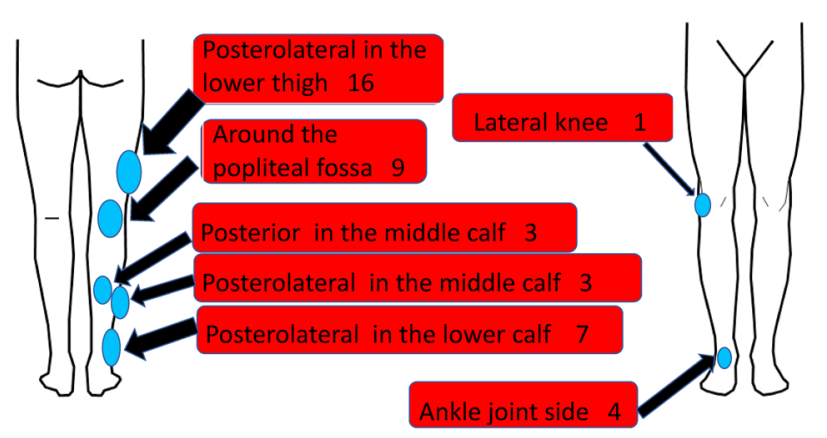

Fig. 1 Distribution of the location of atypical IPVs.

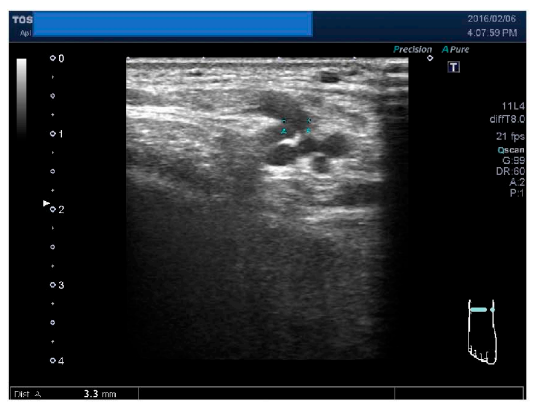

Fig. 2 Ultrasonography of atypical IPVs. ments or muscle heads in the same way as the typical IPV. markedly improved.

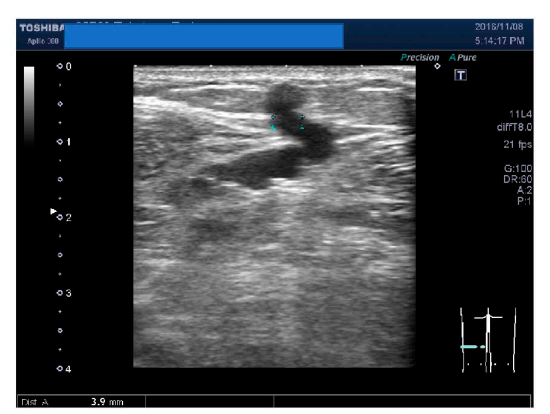

Each IPV passed through the fascia in the area between the muscle compart-

1 month postoperatively for IPVs in the 43 extremities, there were no clear refluxes in all patients; however, 40 extremities were completely blocked under the fascia and for the other 3 patients, to-and-fro blood flow remained at the fascial level, with the patients being followed up while monitoring the development of recurrent varicose veins.

Here, details of a patient are presented. The patient was an 83-year-old woman who had been aware of varicose veins near the right popliteal fossa for more than 30 years; however, because her condition was asymptomatic, she did not seek treatment. The varicose veins had spread over the past 5 years, and the patient had discomfort in the same area and sluggishness and swelling in the lower leg. Physical and lower extremity vein echocardiography findings at initial diagnosis are shown in Figs. 3A and 3B, respectively. The patient was found to have lower extremity varicose veins originating from the IPVs lateral to the hamstring in the lower right thigh and underwent ligation of IPVs and resection of related aneurysms. Surgical findings are presented in Fig. 4. The incompetent perforators were pursued to the fascial level, and all side branches noted till this level were treated. In the findings of the lower extremities (Fig. 5A) and vein echocardiography (Fig. 5B) at 1 month postoperatively, there were no residual varicose veins, incompetent perforators under the fascia were blocked by blood flow, and the symptoms had

Table 1 Symptoms of 36 limbs with atypical incompetent perforating veins without saphenous vein reflux

\begin{tabular}{lc}
\hline Symptoms & Total number \\
\hline Discomfort & 25 \\
Dullness & 14 \\
Edema & 7 \\
Cramps & 6 \\
Dermatitis & 2 \\
Skin ulcer & 1 \\
\hline
\end{tabular}



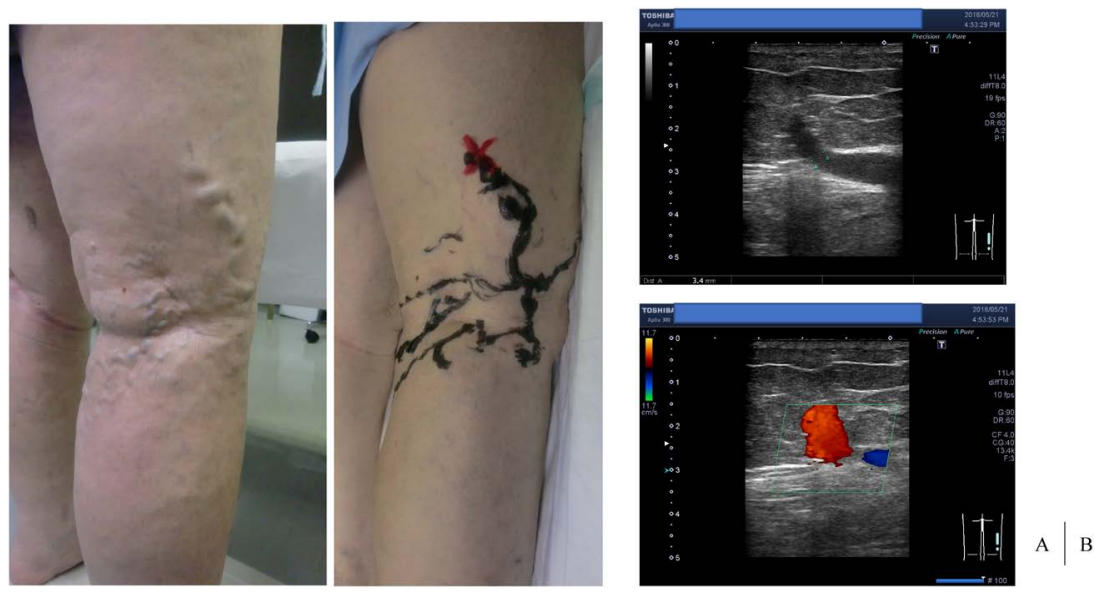

Fig. 3 (A) Preoperative findings of Case 1. The red cross shows the point of the IPV. (B) Venous ultrasonography showing the IPV of Case 1.
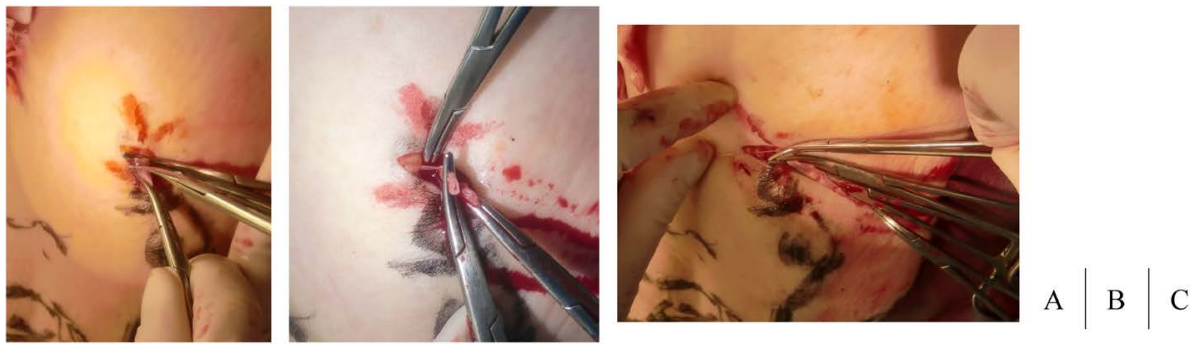

Fig. 4 Operative findings of Case 1.

(A) The IPV is found, peeled off and cut. (B) The IPV is peel off to the central side to the fascia level while processing side branches. (C) Finally, IPV is ligated at the fascia level.
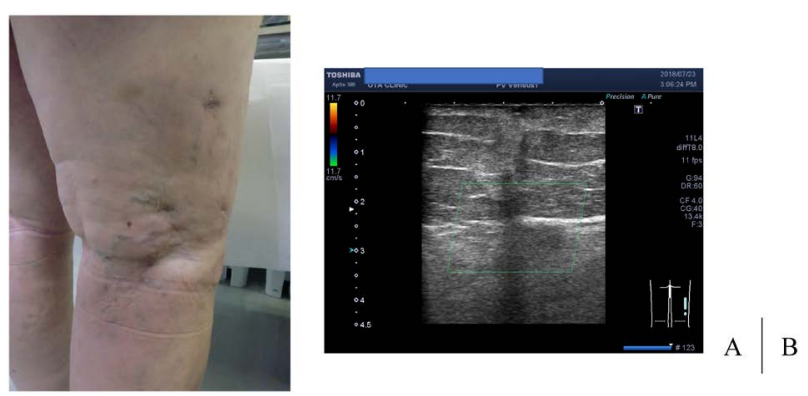

Fig. 5 (A) Findings one month after surgery. (B) The IPV is successfully amputated and there is no blood flow outside the fascia.

\section{Discussion}

The main surgical intervention for lower extremity varicose veins is the treatment for refluxed saphenous veins; in addition to conventional vein stripping, the number of treatments has dramatically increased owing to the availability of insurance coverage for endovascular ablation, and the saphenous vein occlusion using glue will also become a popular treatment alternative in the near future. Moreover, Dodd (UIP name ${ }^{1)}$ : perforating veins of the femoral canal, 5.1.1) and Boyd perforators (UIP name: paratibial perforator veins, 3.1.1) are considered clinically significant because of their association with the great saphenous veins, and Cockett perforators (UIP name: posterior tibial perforator veins, 3.1.2) are considered clinically significant because of their association with lower leg stasis skin lesions. ${ }^{2)}$ In addition to direct ligation and resection, ${ }^{3,4)}$ subfascial endoscopic perforator surgery ${ }^{5-7)}$ and endovascular ablation, ${ }^{8)}$ sclerotherapy, ${ }^{9)}$ and glue treatment, ${ }^{10)}$ which are covered by insurance in Japan, have been reported for these typical perforator refluxes.

On the contrary, approximately 90 perforator branches in the lower extremities have been reported, ${ }^{11)}$ and although atypical IPVs have not gained attention, some reports have analyzed the results of vein echocardiography as part of the pathophysiology referred to as nonsaphenous superficial vein (NSV) reflux, varicose veins without truncal saphenous reflux, and varicose veins with competent saphenous trunks, and it has been suggested that observing not only saphenous veins but also other veins and not overlooking the findings are essential for the accurate treatment of lower extremity varicose veins. ${ }^{12-16)}$ NSV includes not only IPVs but also reflux from the pelvic 
vein and reflux only in the subcutaneous branch, which are associated with different treatment strategies.

Labropoulos et al. previously referred to perforators that were loosely related to the saphenous system, such as those in the lateral or posterior thigh, as "atypical" perforators. ${ }^{17)}$ The analysis of NSV performed by Labropoulos and colleagues ${ }^{12,13)}$ did not include varicose veins due to Cockett's perforator reflux, and although accurate comparisons are difficult because the analysis methods subtly differ in each study, Labropoulos and colleagues have stated that NSV, with the exception of Cockett perforators, was noted in $84(10 \%)$ of 835 limbs with chronic venous insufficiency symptoms, including $19(2 \%)$ in the thigh and 7 (UIP name: popliteal fossa perforating veins, $4.5,1 \%$ ) near the popliteal fossa due to IPV. Although unique classifications of localization of IPVs in the thigh have not been described for each of the numbers listed in the figures, a previous report on a 2,820-limb venous echocardiography series ${ }^{17)}$ described posterolateral thigh perforating veins $(5.4 .3)$ in 24 limbs $(0.85 \%)$. Seidel et al. ${ }^{14)}$ performed vein echocardiography on 1,712 lower limbs suspected of having venous diseases, and a high rate of NSVs was recognized in 735 limbs $(43 \%)$, IPVs in the thigh were observed in $1.3 \%$ and those in the lower leg were observed in $17 \%$, with posterolateral thigh perforating veins based on the UIP name (5.4.3) in 8 limbs $(0.5 \%)$, Cockett perforators (UIP name: posterior tibial perforator vein, 3.1.2) in lower leg IPVs in 76 limbs (4\%), and Boyd perforators (UIP name: paratibial perforator vein, 3.1.1) in 21 limbs $(1 \%)$.

The incidence of atypical IPVs in this study was as high as $12 \%$ for the thighs and lower legs collectively, and although the localization was difficult to match with the UIP name because of the difference in classifications, veins on the posterior surface of the lower legs that were observed in 57 limbs $(3.3 \%)$ were the most prevalent, which may include popliteal fossa perforating veins (4.5). García-Gimeno et al. ${ }^{15)}$ investigated 2036 lower extremity varicose veins originating from reflux using vein echocardiography and found IPVs in 238 limbs (12\%), including Dodd perforators (perforator veins of the femoral canal, 5.1.1) in 85 limbs (4\%), Boyd perforators (paratibial perforator veins, 3.1 .1$)$ in 3 limbs $(0.15 \%)$, and Cockett perforators (posterior tibial perforator veins, 3.1.2) in 10 limbs $(0.5 \%)$. In atypical IPVs, posterolateral thigh perforating veins (5.4.3) were found in 5 limbs $(0.25 \%)$, popliteal fossa perforating veins (4.5) in 21 limbs (1\%), lateral gastrocnemius perforating veins (3.4.2) in 3 limbs $(0.15 \%)$, and anterior ankle perforating veins $(2.2)$ in 1 $\operatorname{limb}(0.05 \%)$. The study by Delis et al. ${ }^{16)}$ revealed that popliteal fossa perforating veins $(4.5)$ were noted in 24 limbs $(3 \%)$ on vein echocardiography of 818 limbs with venous diseases. In each analysis, there was variability in the ratio of IPV occurrence and that of IPV localization.

Although the present study was limited to patients who were surgically treated and cannot be compared with the aforementioned vein echocardiography analyses, in 2,126 limbs for which surgery was simultaneously performed for all lower extremity varicose veins, IPVs were noted in 253 limbs $(12 \%)$, including 27 limbs $(1 \%)$ with Dodd perforators (perforator veins of the femoral canal, 5.1\%), 40 limbs $(2 \%)$ with Boyd perforators (paratibial perforator veins, 3.1\%), and 196 limbs (9\%) with Cockett perforators (posterior tibial perforator veins, 3.1.2). In atypical IPVs, posterolateral thigh perforating veins (5.4.3) were noted in 16 limbs $(0.75 \%)$, popliteal fossa perforating veins $(4.5)$ in 9 limbs $(0.4 \%)$, lateral gastrocnemius perforating veins (3.4.2) in 10 limbs $(0.5 \%)$, and anterior ankle perforating veins $(2.2)$ in 4 limbs $(0.2 \%)$. Among atypical IPVs, posterolateral thigh perforating veins (5.4.3), popliteal fossa perforating veins (4.5), and lateral gastrocnemius perforating veins (3.4.2) were considered significant.

Regarding the presentation of patients with NSV, in the studies by Labropoulos and colleagues ${ }^{12,13)}$ and Seidel et al., ${ }^{14)}$ a higher proportion of multiparous women and fewer patients with stasis skin lesion at $10 \%^{12,13)}$ and $4 \%,{ }^{14)}$ were noted when comparing with saphenous vein type of varicose veins. However, our analysis of atypical IPVs did not show a significant difference from the normal male-to-female ratio of varicose veins in the lower extremities, and $28 \%$ of patients had cutaneous manifestations. This may be related to the observation that patients with NSV include those with pelvic venous origin, some of whom have pelvic stasis syndrome, ${ }^{18)}$ who were excluded from this analysis, and that patients with saphenous vein reflux, who were excluded from the analysis of NSV, are included in this analysis at $17 \%$.

Symptoms associated with NSV were reported in $80 \%$ of patients in the study by Labropoulos and colleagues, ${ }^{12,13)}$ including pain $(72 \%)$, hot flashes $(43 \%)$, and itching $(38 \%)$. In contrast, in our analysis of atypical IPV, discomfort and sluggishness were frequently noted, which may also be attributed to the fact that patients with NSV include those with pelvic venous origin, some of whom have pelvic stasis syndrome. There are few reports on the methods and results of treatment of NSV and atypical IPV, and Labropoulos et al. reported that, although 20 of 24 posterolateral thigh perforators were ligated, reflux remained in 3 branches and required reoperation. ${ }^{17)}$ In this group of patients, in 43 atypical IPVs for ligation and resection, 40 were subfascially closed, whereas 3 exhibited extrafascial stumps with resolved reflux. However, to-andfro blood flow was observed at fascial levels, and the patient was followed up with caution for recurrences. Lower extremity varicose veins associated with atypical IPVs may be treated as side-branch varicose veins in an outpa- 


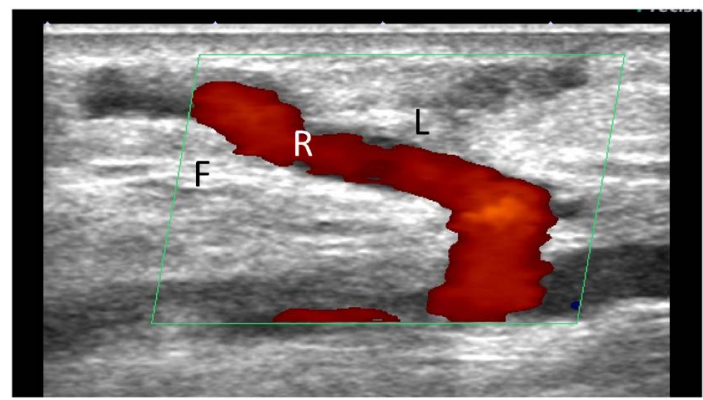

Fig. 6 Recurrence of IPV after direct ligation.

F: fascia; L: ligated IPV; R: recurrent IPV caused by the side branch of the stump outside of the fascia

tient setting and may be conservatively managed without performing lower extremity vein echocardiography, may not be accurately diagnosed when lower extremity vein echocardiography is performed, or may be excluded from surgical treatment if the saphenous vein is intact. The present study shows that surgical treatment of atypical IPVs accounts for $2 \%$ of all lower extremity varicose veins surgeries, and the numbers are not negligible as there are several patients with latency. Although there are several mild cases among the patients presented, some associated aneurysms caused by IPVs are rapidly progressing, and there were few symptomatic patients for whom surgery is indicated. If reflux persists outside the fascia, as shown in Fig. 6, early recurrence may occur from the remnant side branches, and accurate procedures are required. It should be emphasized that this is a technique that can be accurately performed in approximately $10 \mathrm{~min}$ under a $<1$-cm subcutaneous incision if the location is accurately marked using lower extremity vein echocardiography and the three-dimensional attributes over, under, and surrounding the fascia are preliminarily identified. In patients with atypical IPVs as the cause, unlike usual side-branch varicose veins, the IPVs should be stripped and ligated to fascial levels to block blood flow.

\section{Conclusion}

This study clarifies the localization and presentation of patients, symptoms, treatment, and associated results of atypical IPVs other than Dodd, Boyd, and Cockett perforators that are considered clinically significant and presents the importance of treatment based on accurate diagnoses.

\section{Disclosure Statement}

The authors have no conflicts of interest to declare.

\section{Additional Note}

The content of this manuscript was presented on President's request at the 46th Annual Meeting of the Japanese Society for Vascular Surgery in May 2018 in Yamagata, Japan.

\section{References}

1) Caggiati A, Bergan JJ, Gloviczki P, et al. Nomenclature of the veins of the lower limbs: an international interdisciplinary consensus statement. J Vasc Surg 2002; 36: 416-22.

2) Obitsu Y. Anatomy of the peripheral venous system. Journal of Japanese College of Angiology 2009; 49: 195-200. (in Japanese)

3) Kusagawa H, Komada T, Katayama Y, et al. Treatment strategy for incompetent perforating veins. Japanese Journal of Plastic Surgery 2016; 59: 149-56. (in Japanese)

4) Shinozaki K, Ota H, Katayama T, et al. Treatment of incompetent perforating vein of the lower limb using subfascial endoscopic perforator surgery and direct dissection. Japanese Journal of Phlebology 2014; 25: 306-12. (in Japanese)

5) Haruta N, Shinhara R. Subfascial endoscopic perforating vein surgery (SEPS): two-port system subfascial endoscopic perforating vein surgery (TPS-SEPS) with the use of EndoTIP $^{\circledR}$ cannula. Japanese Journal of Phlebology 2011; 22: 63-7. (in Japanese)

6) Matsumoto S, Haruta N, eds. Subfascial Endoscopic Perforator Surgery (SEPS). Tokyo: Tokyo Houki Syuppan, 2016. (in Japanese)

7) Kusagawa H, Haruta N, Shinhara R, et al.; Japanese SEPS study group. Surgical methods and clinical results of subfascial endoscopic perforator surgery in Japan. Phlebology 2018; 33: 678-86.

8) Lawrence PF, Alktaifi A, Rigberg D, et al. Endovenous ablation of incompetent perforating veins is effective treatment for recalcitrant venous ulcers. J Vasc Surg 2011; 54: 737-42.

9) Masuda EM, Kessler DM, Lurie F, et al. The effect of ultrasound-guided sclerotherapy of incompetent perforator veins on venous clinical severity and disability scores. J Vasc Surg 2006; 43: 551-6; discussion, 556-7.

10) Toonder IM, Lam YL, Lawson J, et al. Cyanoacrylate adhesive perforator embolization (CAPE) of incompetent perforating veins of the leg, a feasibility study. Phlebology 2014; 29 Suppl: 49-54.

11) Haruta N. Anatomy of incompetent perforating veins. In: Matsumoto S, Haruta $\mathrm{N}$ eds. Subfascial Endoscopic Perforator Surgery (SEPS). Tokyo: Tokyo Houki Syuppan, 2016: 48-57. (in Japanese)

12) Labropoulos N, Tiongson J, Pryor L, et al. Nonsaphenous superficial vein reflux. J Vasc Surg 2001; 34: 872-7.

13) Malgor RD, Labropoulos N. Pattern and types of nonsaphenous vein reflux. Phlebology 2013; 28 Suppl 1: 51-4.

14) Seidel AC, Miranda F Jr, Juliano Y, et al. Prevalence of varicose veins and venous anatomy in patients without truncal saphenous reflux. Eur J Vasc Endovasc Surg 2004; 28: 38790.

15) García-Gimeno M, Rodríguez-Camarero $S$, Tagarro-Villalba S, et al. Duplex mapping of 2036 primary varicose veins. J 
Vasc Surg 2009; 49: 681-9.

16) Delis KT, Knaggs AL, Hobbs JT, et al. The nonsaphenous vein of the popliteal fossa: prevalence, patterns of reflux, hemodynamic quantification, and clinical significance. J Vasc Surg 2006; 44: 611-9.

17) Labropoulos N, Delis K, Mansour MA, et al. Prevalence and clinical significance of posterolateral thigh perforator vein incompetence. J Vasc Surg 1997; 26: 743-8.

18) Tauchi Y, Yamada M, Okuda N, et al. Experience of endovascular treatment for pelvic congestion syndrome. Japanese Journal of Vascular Surgery 2014; 23: 716-9. (in Japanese) 